\title{
Controlling the Bonding and Band Gaps of Solid Carbon Monoxide with Pressure
}

\author{
Jian Sun, ${ }^{1, *}$ Dennis D. Klug, ${ }^{2}$ Chris J. Pickard, ${ }^{3}$ and Richard J. Needs ${ }^{4, \dagger}$ \\ ${ }^{1}$ Lehrstuhl für Theoretische Chemie, Ruhr-Universität Bochum, 44780 Bochum, Germany \\ ${ }^{2}$ Steacie Institute for Molecular Sciences, National Research Council of Canada, Ottawa, K1A OR6, Canada \\ ${ }^{3}$ Department of Physics and Astronomy, University College London, Gower Street, London WC1E 6BT, United Kingdom \\ ${ }^{4}$ Theory of Condensed Matter Group, Cavendish Laboratory, Cambridge CB3 OHE, United Kingdom
}

(Received 30 September 2010; published 8 April 2011)

\begin{abstract}
We use a combination of a searching method and first-principles electronic structure calculations to predict novel structures of carbon monoxide (CO) which are energetically more stable than the known structures. The most stable forms of $\mathrm{CO}$ at zero pressure consist of metallic polycarbonyl chains with single and double bonds, rather than the familiar triply bonded insulating $\mathrm{CO}$ molecules. At pressures $>2 \mathrm{GPa}$ the most stable phases are semiconducting and insulating singly bonded three-dimensional framework and layered structures. We also find a molecular $\mathrm{Pbcm}$ structure which is more stable than the $R 3 c$ structure proposed previously for the observed $\epsilon$ phase.
\end{abstract}

DOI: 10.1103/PhysRevLett.106.145502

PACS numbers: 61.50.Ks, 71.20.-b, 81.05.Zx, 81.40.Vw

Pressure-induced polymerization of molecular crystals results in materials with entirely new electronic, optical, and physical properties [1,2]. CO is one of the most extensively studied molecular crystals [3-12]. Studies of polymerization of $\mathrm{CO}$ are motivated by the desire to understand the fundamental physics and chemistry of geological and planetary processes, where $\mathrm{CO}$ is present, and to search for new materials [13] exhibiting properties such as "high energy density" [10] and "superhardness" [14,15].

$\mathrm{CO}$ has the strongest chemical bond known and it shows some similarities to molecular nitrogen $\left(\mathrm{N}_{2}\right)$, which has the strongest homopolar bond. $\mathrm{CO}$ and $\mathrm{N}_{2}$ molecules are isoelectronic and isostructural and they are characterized by very strong and short triple bonds. The $\mathrm{CO}$ molecule has a very small dipole moment and it is therefore expected to form structures similar to $\mathrm{N}_{2}$. Molecular phases of $\mathrm{CO}$ and $\mathrm{N}_{2}$ become unstable at high pressures because structures with single and double bonds are in general denser. Polymeric CO is also expected to be a good candidate high-energy-density material, as considerable energy is released on decomposition into $\mathrm{C}$ and $\mathrm{CO}_{2}$ [10-12]. So far, only amorphous polymeric $\mathrm{CO}$ has been found in high pressure experiments.

Liquid CO transforms from the fluid to a disordered hexagonal solid $(\beta-\mathrm{CO})$ at $68.2 \mathrm{~K}$ on cooling at ambient pressure, and freezes into an ordered cubic structure ( $\alpha$-CO, space group $P 2{ }_{1} 3$ ) below $61.5 \mathrm{~K}$ [3]. On compression at room temperature, $\mathrm{CO}$ transforms from the fluid to the solid $\beta$ phase at about $2.5 \mathrm{GPa}$ [4,5], and then into the disordered cubic $\delta$ phase at about $4.8 \mathrm{GPa}$ [16]. In contrast, compression of the liquid at low temperatures results in the solid $\epsilon$ phase whose structure has not been fully resolved, although it has been suggested that it belongs to space group $R 3 c$ [4].

$\mathrm{CO}$ polymerizes at pressures of 4-5 GPa under laser irradiation at temperatures $>80 \mathrm{~K}[3,5]$. The product is recoverable to ambient conditions but is very sensitive to light and moisture. The infrared (IR) absorption of the polymeric material was reported to resemble that of poly-carbon suboxide [17]. Lipp et al. [8] reported that the IR spectrum of the product is substantially different from that of poly-carbon suboxide and might be composed of $-(\mathrm{C}=\mathrm{O})-\mathrm{O}-(\mathrm{C}-)=\mathrm{C}<$ units. Subsequent investigations have concluded that the polymer is based on lactone esters with rings closed by $\mathrm{C}=\mathrm{C}$ bonds $[10,11]$. Ceppatelli et al. [12] investigated the effects of pressure and irradiation and showed that the reaction mechanisms are highly temperature dependent: the amorphous material produced at low temperature $(<300 \mathrm{~K})$ is formed from polycarbonyl chains containing anhydride groups $\left[R-(\mathrm{C}=\mathrm{O})-\mathrm{O}-(\mathrm{C}=\mathrm{O})-R^{\prime}\right]$, while $\mathrm{CO}_{2}$ and epoxy rings are formed at temperatures $\geq 300 \mathrm{~K}$.

Despite these advances, the phase diagram of solid CO is still far from being understood. In this study, a searching scheme [18-20] combined with first-principles densityfunctional theory (DFT) calculations is used to explore structures of $\mathrm{CO}$, revealing several novel phases with very different structural and electronic properties. The DFT calculations were performed using the PerdewBurke-Ernzerhof (PBE) [21] generalized gradient approximation (GGA) density functional. The main calculations were performed with the CASTEP plane-wave code and [22] ultrasoft pseudopotentials. Phonon calculations were performed using DFT perturbation theory and the ABINIT code [23]. Searches were performed at 5, 20, 40, and $100 \mathrm{GPa}$, with $1,2,3$, and $4 \mathrm{CO}$ molecules per cell (not all combinations of these pressures and numbers of molecules were studied). We relaxed up to 1000 initial structures per system. To study the polymeric regime in more detail, we performed extensive searches at $40 \mathrm{GPa}$, using initial structures with 6,8 , or 12 atoms constrained to form polymeric chains with 3 -coordinated $\mathrm{C}$ atoms. Further details 
of the calculations are given in the supplemental material [24].

The enthalpy-pressure relations of the most interesting phases are plotted in Fig. 1. Three new molecular structures, $P b c m, P 2_{1} / c$, and $P 4 / \mathrm{nmm}$ are predicted to be more stable than the proposed $R 3 c$ structure of the $\epsilon$ phase. The most stable of these $(\mathrm{Pbcm})$ is more stable than the $\alpha$ phase at pressures $>0.5 \mathrm{GPa}$, which is lower than the experimental transition pressure between the $\alpha$ and $\epsilon$ phases of about $3.4 \mathrm{GPa}$ [4]. The calculated transition pressure between the $\alpha$ and $R 3 c$ phases is approximately $1 \mathrm{GPa}$, but at this pressure $\mathrm{Pbcm}$ is around $7 \mathrm{meV}$ lower in enthalpy than both of them. The structure of the $\epsilon$ phase has not been fully resolved [4], and our calculations suggest that $\mathrm{Pbcm}$ could be either an additional molecular phase existing between the $\alpha$ and $\epsilon$ phases or a candidate for the $\epsilon$ phase itself.

As shown in Fig. 1(b), polymeric chains $\left(P 2_{1} / m\right.$ and $P n m a)$ are more stable than molecular phases, even at zero pressure. At zero pressure, $P 2_{1} / m$ is around $0.18 \mathrm{eV} / \mathrm{CO}$ lower in energy than the molecular $\alpha$ phase. We have performed calculations with the PBE and B3LYP density functionals both with and without van der Waals dispersion corrections. None of these calculations gives the molecular $\alpha$ phase as the lowest in energy at zero pressure, see the supplemental information [24]. The van der Waals interaction is attractive and is therefore expected to reduce the energies of the denser phases more than the molecular ones. We also calculated the vibrational free energies of the phases within the quasiharmonic approximation, finding that it favors $P 2_{1} / m$ over the $\alpha$ phase at low temperatures

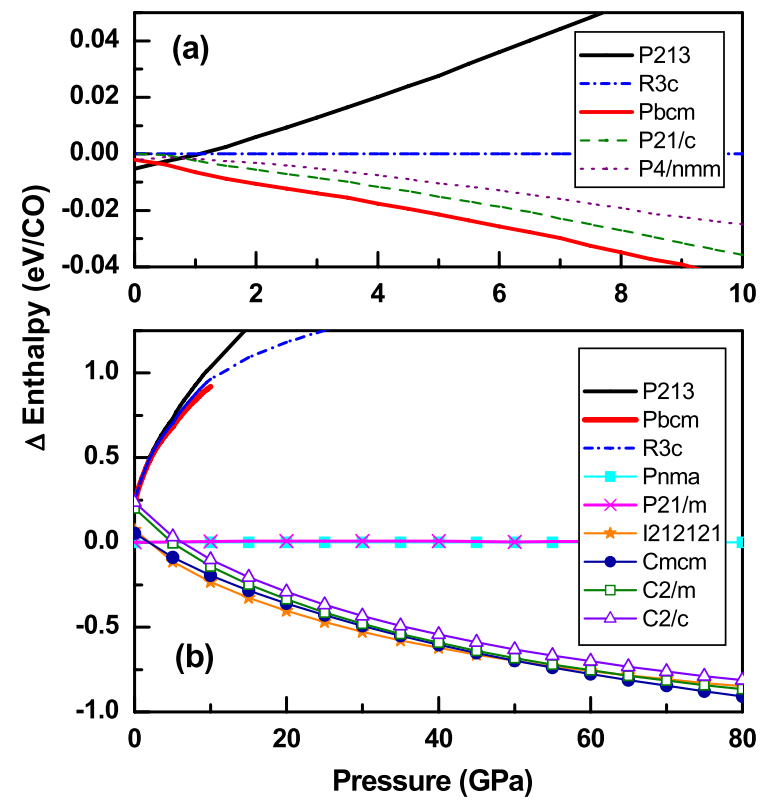

FIG. 1 (color online). Enthalpy-pressure relations for solid CO. (a) Molecular phases relative to $\epsilon-\mathrm{CO}(R 3 c)$. (b) Molecular, polymer and framework or layered structures relative to the $P 2_{1} / \mathrm{m}$ phase.
[24], again supporting our contention that $P 2_{1} / m$ has a substantial region of stability. The $P 2_{1} / m$ phase was not seen in a recent experiment [16], implying that a different synthesis path might be needed, for instance, quenching from the high pressure polymeric phases at low temperature. Alternatively, short polycarbonyl chains [up to $\left.R-(\mathrm{CO})_{5}-R\right]$ have already been synthesized by organic chemists [25], and it is not implausible to imagine that longer chains could be made.

$\mathrm{CO}$ is therefore very different from $\mathrm{N}_{2}$ and $\mathrm{CO}_{2}$ where the molecular crystals are the most stable at ambient pressure. The calculated molecular dissociation pressure leading to cubic gauche $\mathrm{N}$ is about $56 \mathrm{GPa}$ [20] and 20-25 GPa for the $\alpha$ - and $\beta$-cristobalite-like structures of $\mathrm{CO}_{2}$ [26]. $\mathrm{CO}$ polymerizes at very low pressures and is sensitive to light $[5,8,10,12]$, while polymerization of $\mathrm{N}_{2}$ and $\mathrm{CO}_{2}$ is observed at much higher pressures of 110-130 GPa in $\mathrm{N}_{2}$ [15] and 40-80 GPa [14,27,28] in $\mathrm{CO}_{2}$. About $1.0 \mathrm{eV}$ per $\mathrm{CO}(\sim 3.5 \mathrm{~kJ} / \mathrm{g})$ of energy is released when $P 2_{1} / m$ or Pnma $\mathrm{CO}$ decomposes into $\mathrm{C}$ and $\mathrm{CO}_{2}$.

A single-bonded framework structure $\left(I 2_{1} 2_{1} 2_{1}\right)$ is the most stable in the range 2-55 GPa. Other framework structures such as $C 2 / c$ and $C 2 / m$ often appeared in our searches but are less stable than $I 22_{1} 2_{1} 2_{1}$. The singlebonded layered $\mathrm{Cmcm}$ structure is stable at pressures $>55 \mathrm{GPa}$.

The volumes of $\mathrm{Pbcm}$ and the $\alpha$ phase are reduced by about one half on compression to $5 \mathrm{GPa}$. Increasing pressure breaks the $\mathrm{C} \equiv \mathrm{O}$ triple bonds and the adjacent $\mathrm{C}$ atoms form $\mathrm{C}-\mathrm{C}$ bonds, resulting in linear chains which contain a mixture of $\mathrm{C}-\mathrm{C}$ and $\mathrm{C}=\mathrm{O}$ bonds, and the coordination number of the $\mathrm{C}$ atoms increases to three. The lowest-enthalpy $P 2_{1} / m$ and $P n m a$ chain structures are almost degenerate in energy at ambient pressure.

As shown in Fig. 2(b), the $P 2_{1} / m$ chains have the same orientation, while in Pnma they have two orientations and $A B A B$ packing (see the supplemental material [24]). The volume of $P 2_{1} / m$ at $5 \mathrm{GPa}$ is $1 / 3$ smaller than that of $\mathrm{Pbcm}$. Polymeric chains based on a C:O stoichiometry of $1: 1$ were proposed many years ago by Hoffmann and others $[29,30]$. Our structural searches give infinitely long chains, which we expect to be the most stable forms. In the polymerization process, the starting molecular structure contains head-to-tail disorder, and only short polycarbonyl chains tend to form, separated by $\mathrm{C}-\mathrm{O}-\mathrm{C}$ bonds, and the connecting parts form anhydride groups as suggested by recent experiments [12].

Breaking the $\mathrm{C}=\mathrm{O}$ bonds in the chainlike structures results in single-bonded phases including framework and layered structures with fourfold-coordinated $\mathrm{C}$ atoms. As shown in Fig. 2(c), the $I 2_{1} 2_{1} 2_{1}$ framework structure contains five-membered rings consisting of four $\mathrm{C}$ atoms and an $\mathrm{O}$ atom, which are connected by $\mathrm{C}-\mathrm{O}-\mathrm{C}$ bonds. This type of fivefold ring was also seen in an early 
a

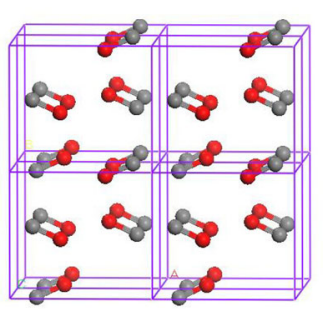

c

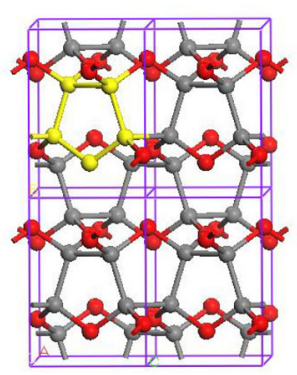

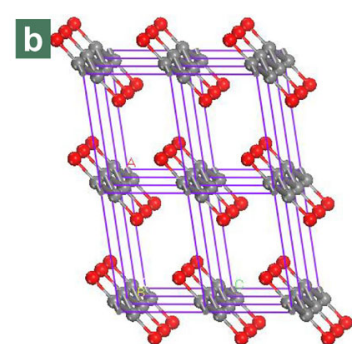

d

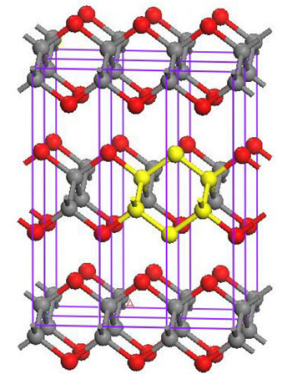

FIG. 2 (color online). Structures of solid CO. (a) $\mathrm{Pbcm}$ (molecular), (b) $P 2_{1} / m$ (chainlike), (c) $I 2_{1} 2_{1} 2_{1}$ (framework), (d) $\mathrm{Cmcm}$ (layered). Dark gray and red (medium gray) balls represent $\mathrm{C}$ and $\mathrm{O}$ atoms, respectively. The five- and sixmembered rings of the $I 2_{1} 2_{1} 2_{1}$ and $\mathrm{Cmcm}$ structures are highlighted in yellow (light gray).

first-principles molecular dynamics study of amorphous $\mathrm{CO}$ [9]. It is interesting that the $I 2_{1} 2_{1} 2_{1}$ structure of $\mathrm{CO}$ is related to the theoretically predicted $P 2_{1} 2_{1} 2_{1}$ framework structure of solid $\mathbf{N}$ [20,31], which also contains helical tubes.

At pressures $>55 \mathrm{GPa}$, layered structures become more stable than framework structures. Different packings of the layers have similar energies, but the packing of $\mathrm{Cmcm}$ is the most efficient. In this structure, all the $\mathrm{C}$ atoms are fourfold coordinated and are connected to two $\mathrm{C}$ and two $\mathrm{O}$ atoms, while two $\mathrm{C}$ and two $\mathrm{O}$ atoms form a six-membered ring. The interlayer distance decreases significantly with pressure while the intralayer structure is hardly affected. Layered structures also appear in the polymerized solids of other molecules, such as $\mathrm{N}_{2}$ [31] and $\mathrm{CO}_{2}$ [26]. Structures with conjugated $\mathrm{C}=\mathrm{C}$ bonds as suggested by experiments $[10,11]$ also appear in our search, but they are higher in energy than the chainlike polymers and purely single-bonded structures.

We investigated the mechanical stability of the structures by calculating their phonon dispersion curves. As shown in Fig. 3, the chainlike $P 2_{1} / m$ structure and the framework $I 2_{1} 2_{1} 2_{1}$ structure are mechanically stable at low pressures. The phonon spectrum of $P 2_{1} / \mathrm{m}$ at ambient pressure is divided into three bands, with $\mathrm{C}=\mathrm{O}$ double bond stretching modes around $1600-1750 \mathrm{~cm}^{-1}$, interchain bending modes at $300-1100 \mathrm{~cm}^{-1}$, and interchain modes below $200 \mathrm{~cm}^{-1}$. Phonon calculations show that the $I 22_{1} 2_{1}$ structure might be quench recovered to ambient
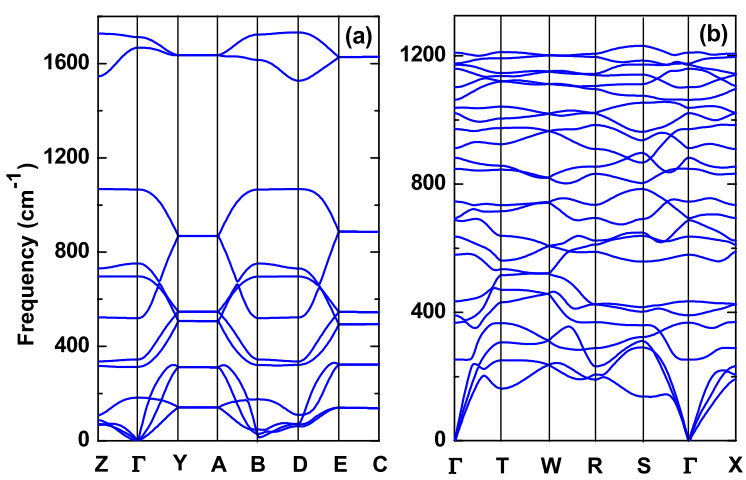

FIG. 3 (color online). Phonon dispersion relations. (a) Polymeric $P 2_{1} / m$ at $0.2 \mathrm{GPa}$ and (b) framework $I 2_{1} 2_{1} 2_{1}$ at $1.0 \mathrm{GPa}$.

pressure at low temperatures. Its bulk modulus at ambient pressure of around $155 \mathrm{GPa}$ is much larger than that of quartz ( $\sim 37 \mathrm{GPa})$. The spread of the phonon frequencies of $I 2_{1} 2_{1} 2_{1}$ is larger than $P 2_{1} / \mathrm{m}$, and they are not separated into distinct groups but have phonon frequencies in the same range as the measured IR spectrum of the amorphous materials [12]. As shown in Fig. 3, Figs. S4 and S6 in the supplemental materials [24], the phonon bands of $P 2_{1} / \mathrm{m}$, $P n m a$ and $\mathrm{Cmcm}$ have very little dispersion along the interchain and interlayer directions, showing that such interactions are weak.

The electronic band structure and density of states (E-DoS) of $P 2_{1} / m$ decomposed into $s$ and $p$ components on the $\mathrm{C}$ and $\mathrm{O}$ atoms are shown in Fig. 4. The molecular $P b \mathrm{~cm}$ phase is insulating but the Pnma and $P 2_{1} / \mathrm{m}$ chainlike structures are metallic. The formation of a metallic polymer at such low pressures is unexpected and is very different from $\mathrm{N}$ which is semiconducting up to at least $240 \mathrm{GPa}$ [32]. The chains within a layer of $P 2_{1} / \mathrm{m}$ are

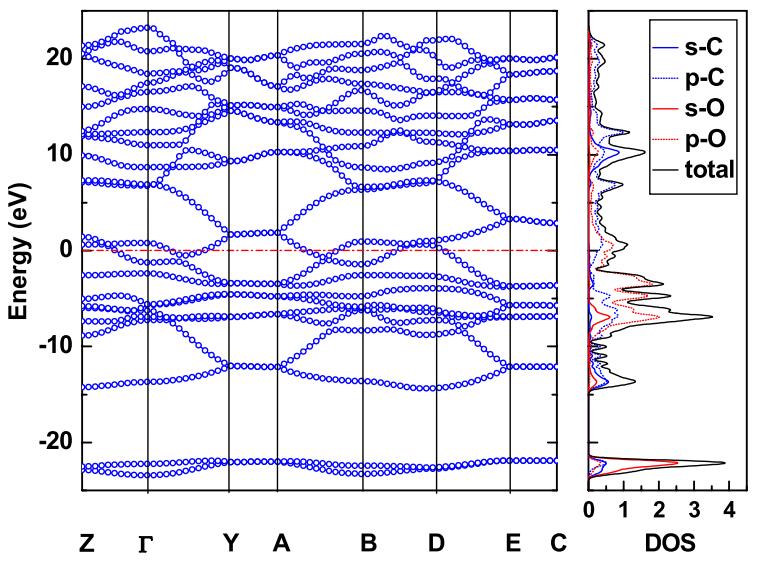

FIG. 4 (color online). Electronic structure of polymeric $P 2_{1} / \mathrm{m}$ at $0.2 \mathrm{GPa}$. The band structure (left) and electronic density of states (right). The zero of energy is at the Fermi level. 
parallel to one another which leads to almost flat bands along the $Y$-A and $E-C$ directions.

As shown in Fig. 4, the E-DoS of the conduction bands arises mainly from the $s$ and $p$ states of the $\mathrm{C}$ atoms, indicating strong $s-p$ hybridization of the threefoldcoordinated $\mathrm{C}$ atoms. The $p$ states of the $\mathrm{C}$ and $\mathrm{O}$ atoms contribute to the E-DoS close to the Fermi level, while the states at the top of the valence bands from -3 to $-10 \mathrm{eV}$ derive mostly from $\mathrm{O} p$ states, and the states from -10 to $-15 \mathrm{eV}$ derive from the $\mathrm{C} s$ and $p$ states and $\mathrm{O} s$ states. The narrow bands around $-22.5 \mathrm{eV}$ derive from the $\mathrm{O} s$ states and give a strongly peaked E-DoS.

The band structure of $I 2_{1} 2_{1} 2_{1}$ at $20 \mathrm{GPa}$ (see the supplemental material [24]) shows insulating behavior with an energy gap of about $4.4 \mathrm{eV}$, and it should therefore be transparent. The band gap of $\mathrm{Cmcm}$ at $80 \mathrm{GPa}$ is calculated to be about $0.5 \mathrm{eV}$ and, considering that the density functional usually tends to underestimate band gaps, this corresponds to semiconducting behavior. The band gap of Cmcm decreases to $\sim 0.1 \mathrm{eV}$ at $150 \mathrm{GPa}$, indicating that pressure substantially affects the band structure of the layered $\mathrm{CO}$ structure, in contrast to $\mathrm{N}$, whose band gaps are much less sensitive to pressure [31].

In summary, we have predicted several new $\mathrm{CO}$ structures to be energetically more stable than the currently known phases. Three molecular phases $\left(P 2_{1} / c\right.$, $P 4 / \mathrm{nmm}$, and $P b \mathrm{~cm}$ ) are more stable at pressures $>0.5 \mathrm{GPa}$ than the ordered $R 3 c$ structure previously proposed for the $\epsilon$ phase, $P b c m$ being the most stable of them. It is energetically favorable for $\mathrm{CO}$ to polymerize even at zero pressure-the most stable forms at zero pressure are found to be polycarbonyl chains, not molecules. The most stable $P 2_{1} / \mathrm{m}$ and Pnma polycarbonyl chains are metallic and contain threefold-coordinated $\mathrm{C}$ atoms, with a mixture of double and single bonds. The single-bonded threedimensional framework $I 2_{1} 2_{1} 2_{1}$ structure and the layered $\mathrm{Cmcm}$ structure are stable at 2-55 GPa and $>55 \mathrm{GPa}$, respectively. The polycarbonyl $P 2_{1} / \mathrm{m}$ phase might be quench recovered from the high pressure polymeric phases at low temperature, or it might be possible to devise an organic synthesis route. The band gaps of the $\mathrm{CO}$ structures can be tuned with increasing pressure from insulating behavior (molecular phases), to metallic $\left(P 2_{1} / \mathrm{m}\right.$ and $P n m a)$, and then back to insulating $\left(I 2_{1} 2_{1} 2_{1}\right)$ and semiconducting $(\mathrm{Cmcm})$. The lowest-enthalpy phase of each bonding type is mechanically stable. We have also identified some of the spectral features, e.g., the "flat phonon bands" that appear in the phonon dispersion curves of the chainlike and layered structures.

J. S. gratefully acknowledges financial support from the Alexander von Humboldt $(\mathrm{AvH})$ foundation. C. J.P. and R. J. N. were supported by the EPSRC. The calculations were carried out at Bovilab@RUB (Bochum) and on the supercomputers at NRC (Ottawa). *jian.sun@theochem.rub.de

†rn11@cam.ac.uk

[1] R. J. Hemley, Annu. Rev. Phys. Chem. 51, 763 (2000).

[2] V. Schettino and R. Bini, Phys. Chem. Chem. Phys. 5, 1951 (2003).

[3] D. T. Cromer, D. Schiferl, R. Lesar, and R. L. Mills, Acta Crystallogr. Sect. C 39, 1146 (1983).

[4] R. L. Mills, D. Schiferl, A. I. Katz, and B. W. Olinger, J. Phys. (Paris) 45, C8-187 (1984).

[5] A. I. Katz, D. Schiferl, and R. L. Mills, J. Phys. Chem. 88, 3176 (1984).

[6] R. L. Mills, B. Olinger, and D. T. Cromer, J. Chem. Phys. 84, 2837 (1986).

[7] P. F. Fracassi, G. Cardini, S. O'Shea, R. W. Impey, and M. L. Klein, Phys. Rev. B 33, 3441 (1986).

[8] M. Lipp, W. J. Evans, V. Garcia-Baonza, and H.E. Lorenzana, J. Low Temp. Phys. 111, 247 (1998).

[9] S. Bernard, G. L. Chiarotti, S. Scandolo, and E. Tosatti, Phys. Rev. Lett. 81, 2092 (1998).

[10] M. J. Lipp, W. J. Evans, B. J. Baer, and C. S. Yoo, Nature Mater. 4, 211 (2005).

[11] W. J. Evans et al., Chem. Mater. 18, 2520 (2006).

[12] M. Ceppatelli, A. Serdyukov, R. Bini, and H. J. Jodl, J. Phys. Chem. B 113, 6652 (2009).

[13] P. F. McMillan, Nature Mater. 1, 19 (2002).

[14] C. S. Yoo et al., Phys. Rev. Lett. 83, 5527 (1999).

[15] M. I. Eremets et al., Nature Mater. 3, 558 (2004).

[16] A. Serdyukov, M. Vetter, A. Brodyanski, and H. J. Jodl, Low Temp. Phys. 36, 424 (2010).

[17] A. W. Snow, H. Haubenstock, and N.L. Yang, Macromolecules 11, 77 (1978).

[18] C. J. Pickard and R. J. Needs, Phys. Rev. Lett. 97, 045504 (2006).

[19] C. J. Pickard and R. J. Needs, Nature Phys. 3, 473 (2007).

[20] C. J. Pickard and R. J. Needs, Phys. Rev. Lett. 102, 125702 (2009).

[21] J. P. Perdew, K. Burke, and M. Ernzerhof, Phys. Rev. Lett. 77, 3865 (1996).

[22] S. J. Clark et al., Z. Kristallogr. 220, 567 (2005).

[23] X. Gonze et al., Comput. Mater. Sci. 25, 478 (2002).

[24] See supplemental material at http://link.aps.org/ supplemental/10.1103/PhysRevLett.106.145502 for details of calculation methods and selected results for $\mathrm{CO}$ structures.

[25] M. B. Rubin and R. Gleiter, Chem. Rev. 100, 1121 (2000).

[26] J. Sun et al., Proc. Natl. Acad. Sci. U.S.A. 106, 6077 (2009).

[27] M. Santoro et al., Nature (London) 441, 857 (2006).

[28] V. Iota et al., Nature Mater. 6, 34 (2007).

[29] R. Hoffmann, Vestn. Moskov. Univ. Ser. Fiz. Astr. 69 (1962).

[30] G. Frapper et al., Chem. Commun. (Cambridge) 2011 (1997).

[31] Y.M. Ma, A.R. Oganov, Z.W. Li, Y. Xie, and J. Kotakoski, Phys. Rev. Lett. 102, 065501 (2009).

[32] M. I. Eremets, R. J. Hemley, H. Mao, and E. Gregoryanz, Nature (London) 411, 170 (2001). 